STRUCTURAL BIOLOGY COMMUNICATIONS

ISSN 2053-230X

\title{
Rv0100, a proposed acyl carrier protein in Mycobacterium tuberculosis: expression, purification and crystallization. Corrigendum
}

\author{
Jasper Marc G. Bondoc, ${ }^{a}$ Hiten J. Gutka, ${ }^{\text {a,b }}$ Mashal M. Almutairi, ${ }^{\text {a,c,d,e }}$ \\ Ryan Patwell, ${ }^{a, f}$ Maxwell W. Rutter, ${ }^{a, g}$ Nina M. Wolf, ${ }^{a}$ Ram Samudrala, \\ Shahila Mehboob, ${ }^{i *}$ Alexey Dementiev, ${ }^{k}$ Cele Abad-Zapatero ${ }^{\mathrm{a}, \mathrm{l}}$ and Farahnaz \\ Movahedzadeh $^{\mathrm{a}, \mathrm{j} ;}$
}

Received 20 February 2020

Accepted 26 February 2020

Edited by R. Sankaranarayanan, Centre for
Cellular and Molecular Biology, Hyderabad,
India

Keywords: acyl carrier protein; Mycobacterium tuberculosis; Mycobacterium smegmatis; Rv0100; corrigendum.

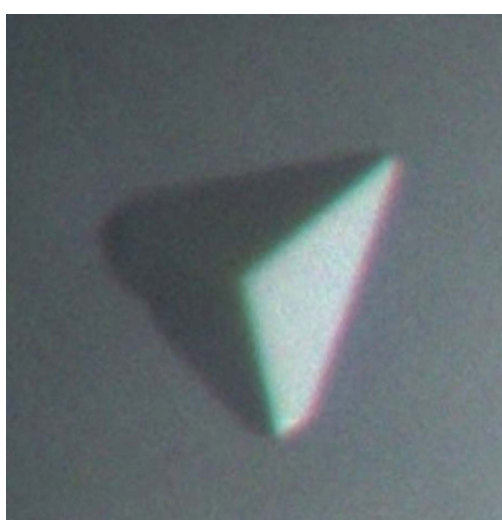

\begin{abstract}
anstitute for Tuberculosis Research, College of Pharmacy, University of Illinois at Chicago, 833 South Wood Street, Chicago, IL 60612, USA, 'b Oncobiologics Inc., 7 Clarke Drive, Cranbury, NJ 08512, USA, ${ }^{\mathbf{c} D e p a r t m e n t ~ o f ~ P h a r m a c o l o g y ~}$ and Toxicology, College of Pharmacy, King Saud University, Riyadh 12371, Saudi Arabia, 'Vaccines and Biologics Research Unit, College of Pharmacy, King Saud University, Riyadh 12371, Saudi Arabia, ${ }^{\mathbf{e}}$ Department of Pediatrics and Molecular Virology and Microbiology, National School of Tropical Medicine, Baylor College of Medicine, 1 Baylor Plaza, Houston, TX 77030, USA, 'Department of Psychiatry, University of Illinois at Chicago, 1601 West Taylor Street, Room 425, Chicago, IL 60612, USA, '⿳亠口冋口⺝ Hollingbery and Son Hops Inc., 302 North First Avenue, Yakima, WA 98907, USA, hepartment of Biomedical Informatics, Jacobs School of Medicine and Biomedical Sciences, State University of New York (SUNY), University at Buffalo, 77 Goodell Street, Buffalo, NY 14203, USA, 'NovaScan Inc., 950 N. 12th Street, Milwaukee,, WI 53233, USA, 'Department of Pharmaceutical Sciences, College of Pharmacy, University of Illinois at Chicago, 833 South Wood Street, Chicago, IL 60612, USA, ${ }^{\mathbf{k}}$ Structural Biology Center, Advanced Photon Source, Argonne National Laboratory, 9700 S Cass Ave, Lemont, IL 60439, USA, and 'Center for Biomolecular Sciences, University of Illinois at Chicago, Chicago, IL 60612, USA. *Correspondence e-mail: shahila@gmail.com, movahed@uic.edu
\end{abstract}

The true identity of the protein found in the crystals reported by Bondoc et al. [(2019), Acta Cryst. F75, 646-651] is given.

As described in the article by Bondoc et al. (2019), attempts to solve the crystal structure ( 2 monomers per a.u. in the $H 3$ cell), using existing structural homologs of Rv0100 Mycobacterium tuberculosis from the Protein Data Bank failed. Structural models obtained from the amino-acid sequence using the I-THASSER protein prediction server also failed. Refinement of tentative solutions obtained by MOLREP and PHASER stalled at $R_{\mathrm{w}}=0.44, R_{\mathrm{f}}=0.51$, even when assuming a lower symmetry $P 1$ lattice with nearly $H 3$ pseudo-symmetry. Detailed examination of the PDB entries related to proteins from Mycobacterium smegmatis, the organism that was used as an expression strain, revealed the existence of entry $3 \mathrm{gwm}$ corresponding to the crystal structure of the holo-[acyl-carrierprotein] synthase (ACPS). This structure was released in 2010 (Poulsen, C., Wilmanns, M. \& Song, Y. H., unpublished work). The crystallographic parameters for this deposition (space group $H 3, a=67.45, b=67.45, c=86.06 \AA, \alpha=\beta=90, \gamma=120^{\circ}$ ) strongly suggested that it was the same protein as the one we had crystallized. Using the protein structure from entry $3 \mathrm{gwm}$, a molecular replacement solution was readily found and was further refined to $R_{\mathrm{w}}=0.19, R_{\mathrm{f}}=0.25$ following standard protocols using the previously reported crystallographic data to $2.0 \AA$ resolution. The amino-acid sequence of the refined protein was fully consistent with the sequence in the ACPS entry, and significant structural differences were found only in the proximity of the three $\mathrm{SO}_{4}$ anions bound in $3 \mathrm{gwm}$ but not in the serendipitously found structure, since there was no sulfate in our crystallization media. The r.m.s.d. between 128 
equivalent $\mathrm{C} \alpha$ carbons in the two structures was $0.58 \AA$ (mean deviation $0.33 \AA$ ).

We hypothesize that our expression in the M. smegmatis strain resulted in the co-purification of ACPS protein from M. smegmatis, as a minor contaminant. This is consistent with the fact that only two very small $(10-20 \mu \mathrm{m})$ crystals were observed in the crystallization droplet and it took about nine months for these crystals to appear. We are certain that the predominant purified protein is Rv0100 from M. tuberculosis confirmed through both mass spectrometry and the fact that the histidine tag was used to isolate the protein. We are confident that the ACPS protein $(\mathrm{MW} \simeq 14000 \mathrm{Da})$ was a minor contaminant that co-purified alongside Rv0100 whose molecular weight is significantly different (11 $122 \mathrm{Da})$.

\section{Funding information}

The following funding is acknowledged: American Lung Association (grant No. RG-82534-N).

\section{References}

Bondoc, J. M. G., Gutka, H. J., Almutairi, M. M., Patwell, R., Rutter, M. W., Wolf, N. M., Samudrala, R., Mehboob, S. \& Movahedzadeh, F. (2019). Acta Cryst. F75, 646-651. 